\title{
Immature Platelet Fraction as A Potential Marker To Differentiate Types of Acute Coronary Syndrome
}

\author{
Endah Indriastuti ${ }^{1}$, Yetti Hernaningsih ${ }^{1}$, Yulia Nadar Indrasari ${ }^{1}$, Andrianto $^{2}$ \\ ${ }^{1}$ Department of Clinical Pathology, Faculty of Medicine, Airlangga University/Dr. Soetomo Hospital, Surabaya, Indonesia. E-mail: \\ endahindriastuti2015@gmail.com \\ 2Department of Cardiology and Vascular Medicine, Faculty of Medicine, Airlangga University/Dr.Soetomo Hospital, Surabaya, Indonesia
}

\begin{abstract}
Acute Coronary Syndrome (ACS) includes ST-Elevation Myocardial Infarction (STEMI), non-ST Elevation Myocardial Infarction (NSTEMI), and Unstable Angina (UA). Platelet plays an essential role in ACS pathogenesis. Immature Platelet Fraction (IPF) and platelet indices can predict platelet activations. Platelet indices consist of platelet count, Mean Platelet Volume (MPV), Platelet Distribution Width (PDW), plateletcrit (Pct). This study aimed to analyze the differences of IPF and platelet indices among ACS patients. This study was an observational analytical cross-sectional study conducted in Dr. Soetomo Hospital during May-September 2019. The subjects consisted of 30-STEMI, 25-NSTEMI, and 24-UA patients. The EDTA-samples were measured for platelet indices and IPF using Sysmex XN-1000. The differences between IPF and platelet indices among STEMI, NSTEMI, and UA patients were analyzed using Kruskal-Wallis and Mann-Whitney test. The IPF values were significantly higher in STEMI patients than NSTEMI and UA patients. The IPF values of NSTEMI patients were higher than UA patients. The MPV, PDW, and P-LCR were significantly higher in STEMI and NSTEMI compared to UA. The MPV, PDW, and P-LCR values of NSTEMI patients were significantly higher than UA patients. The significant differences between STEMI and NSTEMI toward UA might be caused by the more severe thrombotic conditions in myocardial infarction patients than UA. The IPF values were significantly different among each type of ACS patients gave an opportunity using this parameter to differentiate the ACS types. The MPV, PDW, and P-LCR were significantly higher in myocardial infarction patients than UA patients, which also allowed them to use those parameters to differentiate both conditions.
\end{abstract}

Keywords: Acute coronary syndrome, platelet indices, IPF, STEMI, NSTEMI, unstable angina

\section{INTRODUCTION}

Coronary Heart Disease (CHD) is the leading cause of disability and death worldwide. Acute Coronary Syndrome (ACS) is the most common manifestation of Coronary Heart Disease (CHD). It was estimated that CHD caused about 7.4 million deaths in 2015. ${ }^{1}$ Prevalence of CHD in Indonesia was about $0.5 \%$ ( 883,447 people) in 2013 , with a mortality rate of 150 per 100.000 people per year. ${ }^{2}$ This mortality rate was higher than in other countries, such as Thailand and Malaysia. ${ }^{3}$

An acute coronary syndrome is classified into ST-Elevation Myocardial Infarction (STEMI), non-ST-Segment Elevation Myocardial Infarction (NSTEMI), and Unstable Angina (UA). The two former conditions are further called as Myocardial Infarction (MI). There is an elevation of biochemical markers values for myocardial necrosis in MI, but there is no elevation found in UA. ${ }^{4}$

An acute coronary syndrome is commonly initiated by damaged atherosclerotic plaque in the coronary artery. The thrombogenic material inside atherosclerotic plaque will be exposed to the blood circulation. Platelet activation, initiation of the coagulation cascade, and formation of a mural thrombus will be initiated, resulting in heart vessels blocking. A total coronary artery occlusion can cause STEMI. In contrast, partial occlusion or an occlusion in the presence of collateral circulation results in NSTEMI or UA. ${ }^{5,6}$ Myocardial infarction patients have a higher mortality risk. They need early recognition and intervention for re-vascularization and save the living myocards left. ${ }^{7}$

Platelets play essential roles in ACS pathogenesis. Platelets play roles in the formation of atherosclerosis plaque and thrombus after the rupture of atherosclerotic plaque. Platelet consumption in an atherosclerotic blood vessel can stimulate the release of larger platelet by the bone marrow. Larger platelets were metabolically and more active enzymatically as they contain more prothrombotic materials such as thromboxane A2 and express more glycoprotein IIb/IIIa. ${ }^{8,9}$ Advanced 
platelet activation and recruitment occur in thrombus formation after rupture or erosion of atherosclerotic plaque. ${ }^{8-10}$

Platelets can be released from bone marrow as immature platelets. The immature platelet size is usually larger than the mature platelet. The release of immature platelets from bone marrow will increase in response to high platelet turn over; therefore, immature platelet parameters can reflect platelet activation and consumption. Acute coronary syndrome pathogenesis involves platelet activation and stimulation. ${ }^{11}$ Previous studies have found an increase in immature platelets in ACS and MI patients than normal control. ${ }^{11,12}$

Immature platelet fraction is a parameter that reflects the proportion of immature platelet compared to the total platelet. The presence of immature platelet can also influence platelet indices commonly found in routine Complete Blood Count (CBC) test, i.e., platelet count, Mean Platelet Volume (MPV), Platelet Distribution Width (PDW), plateletcrit (Pct), and Platelet-Large Cell Ratio (P-LCR).

Those platelet parameters are considered simple and inexpensive parameters, usually measured during a routine blood test. Therefore, the IPF and other platelet indices can be expected as markers to differentiate types of ACS.

\section{METHODS}

The current study was an observational study conducted in Dr. Soetomo Hospital from May 2019 until September 2019. This research involved 79 patients presenting typical chest pain of acute coronary syndrome patients within 24 hours. Based on clinical manifestations, electrocardiogram results, and cardiac biomarker measurements, the patients were further classified into 30 STEMI patients, 25 NSTEMI patients, and $24 \mathrm{UA}$ patients.

Patients with comorbidities that could affect platelet indices or IPF values such as dengue infection, sepsis, pneumonia, and patients with a myeloproliferative disorder, were excluded. Patients commencing antiplatelet and or anticoagulant therapy were also excluded from this study. The ethical committee approved this study of Dr. Soetomo Hospital, Surabaya, Indonesia, with the number of 1216/KEPK/V/2019. Written informed consent was taken from all study subjects.

Blood samples were collected in Dipotassium Ethylenedinitrotetraacetic acid (EDTA) tubes before antiplatelet or anticoagulant therapy. Demographic data, including age, gender, and risk factors, i.e., smoking habits, hypertension, and Diabetes Mellitus
(DM) history, were obtained. The IPF, MPV, PDW, Pct, $\mathrm{P}-\mathrm{LCR}$, and platelet count values were measured using Sysmex XN-1000 less than four hours after vein punction.

All data were statistically analyzed using SPSS software. Data normality was analyzed using the Shapiro-Wilk test. The differences of IPF, MPV, PDW, Pct, P-LCR, and platelet count values among STEMI, NSTEMI, and UA patients would be analyzed using ANOVA and LSD test if data were normally distributed, otherwise would be analyzed using Kruskal-Wallis and Mann-Whitney test. A Chi-square test was used to compare categorical variables, i.e., smoking habits, hypertension, and diabetes history. A p-value $<0.05$ was considered significant.

\section{RESULTS AND DISCUSSION}

A total of 79 patients were involved and further divided into three groups, i.e., 30 STEMI patients, 25 NSTEMI patients, and 24 UA patients. The total samples used in this study already fulfilled the minimum sample size estimated beforehand. The characteristics of the subjects can be seen in Table 1 . The mean age was $57.9 \pm 11.79$ years old. Brown-Forsythe test showed no statistical difference in age characteristics among groups. Age is one of the unmodifiable risk factors of ACS. The risk of ACS increases with age. ${ }^{13}$

Male dominated the ACS patient subjects. There were $62 \%$ male and $38 \%$ female subjects. Similar results were found in previous studies. ${ }^{10-16}$ These results could be associated with protective estrogen effects toward cardiovascular disease, especially in the pre-menopause period. Estrogen has protective effects on the endothelium by increasing Nitric Oxide (NO) and prostacyclin production, which further have vasodilatation effects. Estrogen also has anti-inflammation effects, increases endothelium regeneration and High-Density Lipoprotein (HDL) levels, also to decrease Low-Density Lipoprotein (LDL) levels. ${ }^{17,18}$

The other ACS risk factors, such as DM, hypertension, and smoking habit, were also found in most study subjects. The Chi-Square test showed no significant difference in gender and risk factors among the three groups. Similar results were also found in a previous study. ${ }^{16}$ The correlation between smoking habits and ACS incidence had been confirmed in several studies. ${ }^{19,20}$ Previous studies showed that chemical substances in the cigarettes such as nicotine and combustion of the cigarettes that release carbon monoxide were oxidants that increase vessel susceptibility of LDL accumulations. ${ }^{21}$ 
The normality test of all variables showed abnormal data distribution; therefore, so the Kruskal-Wallis test was used for statistical analysis. The Mann-Whitney test was then used after any significant difference in the Kruskal-Wallis test was found. The Kruskal-Wallis test showed significant differences in IPF, MPV, PDW, and P-LCR values among the three groups. However, there was no significant difference in platelet counts and Pct parameters among the three groups (Table 2). A further statistical test was conducted toward those four significant parameters.

Table 1. Characteristics of subjects

\begin{tabular}{|c|c|c|c|c|c|}
\hline & \multicolumn{3}{|c|}{ Groups } & \multirow[b]{2}{*}{$\begin{array}{c}\text { Total } \\
(n=79)\end{array}$} & \multirow[b]{2}{*}{ p-value } \\
\hline & $\begin{array}{c}\text { STEMI } \\
(\mathrm{n}=30)\end{array}$ & $\begin{array}{l}\text { NSTEMI } \\
(\mathrm{n}=25)\end{array}$ & $\begin{array}{c}\text { UA } \\
(n=24)\end{array}$ & & \\
\hline \multicolumn{6}{|l|}{ Age (years old) } \\
\hline Mean ( \pm standard deviation) & $\begin{array}{r}60.8 \\
( \pm 11.62)\end{array}$ & $\begin{array}{c}57.9 \\
( \pm 8.61)\end{array}$ & $\begin{array}{c}54.5 \\
( \pm 14.15)\end{array}$ & $\begin{array}{c}57.9 \\
( \pm 11.79)\end{array}$ & $0.150 *$ \\
\hline Gender & & & & & $0.513^{* *}$ \\
\hline Male & 21 (70\%) & $14(56 \%)$ & $14(58.3 \%)$ & 49 (62\%) & \\
\hline Female & $9(30 \%)$ & $11(44 \%)$ & 10 (41.7\%) & 30 (38\%) & \\
\hline \multicolumn{6}{|l|}{ Risk factors } \\
\hline DM & $14(46.7 \%)$ & $15(60 \%)$ & $9(37.5 \%)$ & 38 (48.1\%) & $0.283^{* *}$ \\
\hline Hypertension & $19(63.3 \%)$ & 15 (60\%) & 13 (54.2\%) & 47 (59.5\%) & $0.791^{\star *}$ \\
\hline Smoking & $20(66.7 \%)$ & 11 (44\%) & $13(54.2 \%)$ & 44 (55.7\%) & $0.238^{* *}$ \\
\hline
\end{tabular}

*: Brown-Forsythe test; **: Chi-Square test

Table 2. Immature platelet fraction and platelet indices values among the three groups

\begin{tabular}{ccccc}
\hline Variable & Groups & N & Median (range) & p-value $^{*}$ \\
\hline Platelet count $\left(\times 10^{3} / \mu L\right)$ & STEMI & 30 & $264(127-512)$ & \\
& NSTEMI & 25 & $264(162-428)$ & 0.264 \\
UPV (fL) & UA & 24 & $307(164-596)$ & \\
& STEMI & 30 & $10.6(8.8-13.2)$ & $<0.001$ \\
NSTEMI & 25 & $10.1(8.8-12.5)$ & \\
PDW (fL) & UA & 24 & $9.6(8.2-12.2)$ & \\
& STEMI & 30 & $11.95(9.6-20.2)$ & \\
& NSTEMI & 25 & $11.7(9.4-16.9)$ & \\
Pct (\%) & UA & 24 & $10.3(8.3-14.2)$ & \\
& STEMI & 30 & $0.3(0.17-0.58)$ & \\
& NSTEMI & 25 & $0.31(0.20-1.33)$ & \\
P-LCR (\%) & UA & 24 & $0.29(0.22-0.51)$ & \\
& STEMI & 30 & $28.45(16.0-51.4)$ & \\
IPF (\%) & NSTEMI & 25 & $26.4(15.5-45.5)$ & $<0.001$ \\
& UA & 24 & $21.4(11.2-39.2)$ & \\
& STEMI & 30 & $3.8(1.8-11.9)$ & \\
& NSTEMI & 25 & $2.7(0.8-10.5)$ & \\
& UA & 24 & $2.0(0.5-3.7)$ & \\
\hline
\end{tabular}

* Kruskal-Wallis test

Table 3. IPF, MPV, PDW, and P-LCR values between each group

\begin{tabular}{lccc}
\hline \multirow{2}{*}{ Variable } & \multicolumn{3}{c}{ p-value $^{*}$} \\
\cline { 2 - 4 } & STEMI- NSTEMI & NSTEMI-UA & STEMI-UA \\
\hline IPF & 0.013 & 0.004 & $<0.001$ \\
MPV & 0.078 & 0.002 & $<0.001$ \\
PDW & 0.272 & $<0.001$ & $<0.001$ \\
P-LCR & 0.331 & $<0.001$ & $<0.001$ \\
\hline
\end{tabular}

* Mann-Whitney test 
The median of IPF values of STEMI, NSTEMI, and UA patients showed a gradual decrease of $3.8 \%$, $2.7 \%$, and $2.0 \%$, respectively. The IPF values in all groups can be seen in Table 2. Mann-Whitney test showed statistically significant differences between STEMI and NSTEMI groups, STEMI and UA groups, and NSTEMI and UA, with a p-value of 0.013 , $<0.001$, and 0.004 , respectively. The result of the Mann-Whitney test can be seen in Table 3.

The results of this study were consistent with the previous study's results. Grove et al. found the IPF of non-ST Segment Elevation Acute Coronary Syndrome (NSTE-ACS) patients were significantly different from STEMI patients. ${ }^{12}$ However, that study did not differentiate the IPF values of NSTEMI patients from STEMI patients or UA patients from STEMI patients. Huang et al. found that IPF values of ACS patients were higher than non-ACS patients. ${ }^{22}$

A recent study found that the median of IPF values was proportional to ACS severity. Acute coronary syndrome is a spectrum of conditions that consist of STEMI, NSTEMI, and UA. The seriousness of STEMI and NSTEMI is higher than UA because the occlusions in MI are more severe than UA in two former conditions. On the other hand, the severity of STEMI is higher than NSTEMI as the occlusions in STEMI are total occlusions. ${ }^{5,9}$

Platelet activation can occur before ACS occurrence. Rapid platelet consumption during ACS will stimulate megakaryocyte, causing the release of new platelets by the bone marrow. The release of the new platelets will increase immature platelet count and consequently will increase the IPF value. Immature platelet fraction was known to correlate with MPV; however, large platelet is not always immature platelet and vice versa. Immature platelet fraction has a better ability to reflect immature platelet production released from bone marrow compared to MPV. ${ }^{22}$ This study found significant differences of IPF values in each ACS conditions that these results give the possibility for the IPF parameter to differentiate the three ACS conditions and predict the prognosis of ACS patients through further study.

Platelet indices measurement was conducted in 79 samples. There were significant differences in MPV, PDW, and P-LCR values between STEMI and UA patients. There were a significant difference in MPV, PDW, and P-LCR values between NSTEMI and UA patients. Contrastingly, there were no significant differences in MPV, PDW, and P-LCR values between STEMI and NSTEMI patients. However, the MPV, PDW, and P-LCR values showed a gradation decrease that the values were higher in STEMI patients, followed by NSTEMI and UA patients. The MPV, PDW, and P-LCR values of the three groups can be seen in Table 2. The $p$-values of all parameters can be seen in Table 2 and Table 3.

Statistically, the difference of platelet counts and Pct parameters was not found among the three groups. The platelet count and Pct parameters were not significantly different in the Kruskal-Wallis test that the Mann-Whitney test was not used in these two parameters. The platelet count and Pct values of the three groups are shown in Table 2.

This study found a significant difference in MPV value between STEMI and UA patients also between NSTEMI and UA patients. The MPV value medians in three groups were also found in correlation with ACS severity. Similar results were also found in a previous study. ${ }^{15}$ Mean platelet volume value in MI patients was also influenced by Double Vessel Disease (DVD), or Triple Vessel Disease (TVD) detected using coronary angiography. The involvement of more vessels led to higher MPV value. ${ }^{23}$ Recent study did not evaluate the thrombosis severity of the patients using coronary angiography. The unknown DVD and TVD state of the patients, limitations in this study, might affect the MPV value results among STEMI and NSTEMI patients.

There was a significant difference in platelet distribution width between STEMI and UA groups between NSTEMI and UA groups in this study. The PDW value was higher in STEMI, followed by the NSTEMI group and UA groups. This study result was consistent with previous study results. Platelet distribution width values of MI patients were higher than UA patients and healthy control. ${ }^{14,15,24}$ The increase of PDW correlated with an increase of platelet production and activity in thrombosis. ${ }^{25}$ Platelet distribution width was known to have a positive correlation with MPV. The MPV value in AMI was also affected patient in DVD or TVD condition. The exact thrombosis severity in patients was not known because the coronary angiography was not carried out.

This study found that P-LCR was significantly different between STEMI and UA group and between NSTEMI and UA groups with tiered value, higher in STEMI, followed by NSTEMI, and UA. The previous study result showed that P-LCR-value was more elevated in STEMI and NSTEMI than UA patients, although statistically insignificant. ${ }^{15}$ Khandekar et al. found P-LCR was higher in ACS patients than stable coronary artery disease patients and healthy control. Still, it failed to find any difference in P-LCR-value between AMI and UA patients. ${ }^{26}$ There was a positive correlation between MPV and PDW and MPV and 
P-LCR values. ${ }^{27}$ Recent study found significant differences in P-LCR values between STEMI and UA, also between NSTEMI and UA patients. This study's significant result could be affected by a better sample proportion and a larger sample number than the previous research.

There was no significant difference in platelet count; however, there was a decreased platelet count in STEMI and NSTEMI compared to UA patients (Table 2). This result was consistent with other previous studies. ${ }^{9,2426}$ Recent and previous study results showed a platelet role in ACS pathogenesis, in which platelet will be consumed. Platelet count in steady-state conditions will be affected by various factors, such as genetic, age, and gender, leading to different platelet counts among individuals. ${ }^{28}$ This result showed another factor and platelet count, which influences ACS, although platelet counts play a role in ACS.

This study also failed to find any significant difference in PCT among three ACS groups. The previous studies conducted by Al-Obeidi et al., Pipliwal et al., and Costa et al. also found similar results with this recent study. ${ }^{24,29,30}$ Plateletcrit value is a platelet parameter, which is influenced by platelet count. The inconsistent and insignificant result of PCT in this study and in a previous study might be correlated with the wide variance of platelet count among individuals. Therefore, the role of PCT in differentiating ACS conditions remains unclear.

\section{CONCLUSION AND SUGGESTION}

Recent study results allowed the IPF parameter to differentiate the three ACS conditions and predict ACS patients' prognosis through further study. On the other hand, the MPV, PDW, and P-LCR values were able to differentiate MI and UA. Further research, which measures thrombotic severity using coronary angiography, was needed for a better result.

\section{REFERENCES}

1. Jayaraj JC, Davatyan K, Subramanian SS, Priya J. Epidemiology of myocardial infarction. Intech Open, 2018; 10-17.

2. Badan Penelitian dan Pengembangan Kesehatan Kementerian Kesehatan RI. Riset kesehatan dasar. Jakarta, Kementerian Keseharan Republik Indonesia. 2013; 91-92.

3. Hata J, Kiyohara Y. Epidemiology of stroke and coronary artery disease in Asia. Circ J, 2013; 77 : 1923-1932.

4. Thygesen K, Alpert JS, Jaffe AS, Simoons ML, Chaitman
$\mathrm{BR}$, et al. Third universal definition of myocardial infarction. Circulation, 2012; 126: 2020-2035.

5. Anderson JL, Morrow DA. Acute myocardial infarction. N Engl J Med, 2017; 37: 2053-2064.

6. Reed GW, Rossi J, Cannon CP. Acute myocardial infarction. Lancet, 2017; 389: 197-210.

7. Kumar A, Cannon CP. Acute coronary syndromes: Diagnosis and management, part I. Mayo Clin proc, 2009; 84(10): 917-938.

8. Slavka G, Perkmann T, Haslacher H, Greisenegger S, Marsik C, et al. Mean platelet volume may represent a predictive parameter for overall vascular mortality and ischemic heart disease. Arterioscler Thromb Vasc Biol, 2011; 31(5): 1215-1228.

9. Syahrir W, Kurniawan LB, Rauf D. Analysis of mean platelet volume as a marker for myocardial infarction and non-myocardial infarction in acute coronary syndrome. Indonesian Journal of Clinical Pathology and Medical Laboratory. 2017; 24(1): 76-80.

10. Patil KS, Karchi SD. A comparative study of platelet indices in acute coronary syndrome. International Journal of Contemporary Medical Research, 2017; 4(3): 657-660.

11. Khalifa KA, Helwa MA, Mohammed MAM. Reticulated platelets in acute coronary syndrome patients. Menoufia Med J, 2017; 30: 880-886.

12. Grove EL, Hvas A, Kristensen SD. Immature platelet in patients with acute coronary syndrome, 2009; 101: 151-156.

13. Dhingra R, Vasan RS. Age as a cardiovascular risk factor. Med Clin North Am, 2012; 96(11): 87-91.

14. Alvitagala BY, Azra MAF, Kottahachchi DU, Jayasekera MMPT, Wijesinghe RANK. A study of association between platelet volume indices and ST-elevation myocardial infarction. IC Heart \& Vasculature, 2018; 21:7-10.

15. Machanda J, Potekar RM, Badiger S, Tiwari A. The study of platelet indices in acute coronary syndromes. Annals of Pathology and Laboratory Medicine. 2015; 2(1): A30-A35.

16. Muhibbah, Wahid A, Agustina R, Illiandri $\mathrm{O}$. Karakteristik pasien sindrom koroner akut pada pasien rawat inap ruang Tulip di RSUD Ulin Banjarmasin. Indonesian Journal for Health Science, 2019; 3(1): 6-12.

17. Chakrabarti S, Morton JS, Davidge ST. Mechanism of estrogen on endothelium: An overview. Canadian Journal of Cardiology, 2013; 13:1-8.

18. Guetta V, Cannonll RO. Cardiovascular effects of estrogen and lipid-lowering therapies in postmenopausal women. Circulation, 1996; 93: 1928-1937.

19. Rosengren A, Wallentin L, Simoons M, Gitt AK, Behar S, et al. Cardiovascular risk factors and clinical presentation in acute coronary syndromes. Heart, 2005; 91(9): 1141-1147.

20. Ceponiene I, Zaliaduonyte-Peksiene D, Gustiene O, Tamosiunas A, Zaliunas R. Association of major cardiovascular risk factors with the development of acute coronary syndrome in Lithuania. Eur Heart J Suppl, 2014; 16(Suppl A): A80-A83. 
21. Diastutik D. Proporsi karakteristik penyakit jantung koroner pada perokok aktif berdasarkan karakteristik merokok. Jurnal Berkala Epidemiologi, 2016; 4(3): 326-337.

22. Huang $\mathrm{HL}$, Chen $\mathrm{CH}$, Kung $\mathrm{CT}$, Sung $\mathrm{PH}$, You $\mathrm{HL}$, et al. Clinical utility of mean platelet volume and immature platelet fraction in acute coronary syndrome. Biomed J, 2019; 42(2): 107-115.

23. Ahamed $H$, Henry RA, Pai R. Association of mean platelet volume and acute coronary syndrome. Int J Res Med Sci, 2017; 5(4): 1217-1220.

24. Pipliwal PS, Singh G, Ishran R, Bansal S. Mean platelet volume and other platelet volume indices in patients with acute myocardial infarction: A case-control study. IOSR-JDMS, 2015; 14(11): 35-38.

25. Cetin MS, Cetin EHO, Akdi A, Aras D, Topaloglu S, et al. Platelet distribution width and plateletcrit: Novel biomarkers of ST-elevation myocardial infarction in young patients. Kardiologia Polska, 2017; 75 (10): 1005-1012.

26. Khandekar MM, Khurana AS, Deshmukh SD, Kakrani $A L$, Katdare $A D$, et al. Platelet volume indices in patients with coronary artery disease and acute myocardial infarction: An Indian scenario. J Clin Pathol, 2006; 59: 146-149.

27. Ranjith MP, Divya R, Mehta VK, Krishnan MG, KamalRaj $R$, et al. Significance of platelet volume indices and platelet count in ischaemic heart disease. J Clin Patho, 2009; 62: 830-833.

28. Daly M. Determinant of platelet count in humans. Haematologica, 2011; 96(1): 10-13.

29. Al-Obeidi SRH, Ahmedm SH, Obeid FA. Evaluation of platelet indices in patients with acute coronary syndrome. Muntansiriya Medical Journal, 2013; 12(1): 58-63.

30. Costa SC, Vinagre CGM, Chacra APM, Azevedo MRA. Platelet indices in patients with acute coronary syndrome. Journal of Biosciences and Medicines, 2015; 3: 71-76. 